Research Article

\title{
Dynamic Spectrum Pricing with Secondary User's Normal Demand Preference
}

\author{
Li Wang $\mathbb{D}^{1}$ and Feng $\mathrm{Li} \mathbb{D}^{2}$ \\ ${ }^{1}$ College of Information Engineering, Zhejiang University of Technology, Hangzhou, Zhejiang 310023, China \\ ${ }^{2}$ School of Electronic Science and Engineering, Nanjing University, Nanjing, Jiangsu 210023, China \\ Correspondence should be addressed to Feng Li; fenglzj@zjut.edu.cn
}

Received 15 September 2017; Revised 30 November 2017; Accepted 17 December 2017; Published 1 March 2018

Academic Editor: Mariusz Glabowski

Copyright (C) 2018 Li Wang and Feng Li. This is an open access article distributed under the Creative Commons Attribution License, which permits unrestricted use, distribution, and reproduction in any medium, provided the original work is properly cited.

During secondary user's dynamic access to authorized spectrum, a key issue is how to ascertain an appropriate spectrum price so as to maximize primary system's benefit and satisfy secondary user's diverse spectrum demands. In this paper, a scheme of pricing-based dynamic spectrum access is proposed. According to the diverse qualities of idle spectrum, the proposed scheme applies a Hotelling game model to form the spectrum pricing problem. Firstly, establish a model of spectrum leasing, among which the idle spectrum with different qualities constitutes a spectrum pool. Then, divide the idle spectrum into equivalent width of leased channels, which will be uniformly sold in order. Secondary users can choose proper channels to purchase in the spectrum pool according to their spectrum usage preferences which are subject to normal distribution and affected by the spectrum quality along with market estimation. This paper analyzes the effect of spectrum pricing according to the primary system's various tendencies to spectrum usage and economic income. Numerical results evaluate the effectiveness of the proposed pricing method in improving the primary system's profits.

\section{Introduction}

With the rapid development of wireless communication technology and the establishment of next-generation $5 \mathrm{G}$ communication standard, high-quality idle spectrum is more scarce which has become one of the bottlenecks restricting the development of wireless communication technology [1-4]. Cognitive radio which is based on dynamic spectrum access has attracted more and more attention of academy and engineering in recent years $[5,6]$. Various kinds of emerging network technologies have begun to adopt dynamic spectrum detection and dynamic spectrum access to improve the efficiency of spectrum utilization. In the process of dynamic spectrum access, primary users owning licensed spectrum can lease the idle channels to secondary users to gain incomes. For primary users, how to identify an optimal channel pricing to maximize its own profit has become a significant issue. In this paper, we directly price the idle spectrum of authorized users according to the secondary user's diverse preferences. The spectrum pricing scheme has a prior estimate to the spectrum market. Compared with the spectrum auction, it does not need many overheads and improves the convenience of the spectrum access.

Spectrum trading provides an efficient way for secondary users to dynamically access licensed bands while the financial gains can encourage primary users to lease unused spectrum temporarily. Generally, the participants can perform the deal by auction-based method or pricing-based method. The spectrum auction mechanism can be divided into many kinds according to different application circumstances, such as trust-based auction which relaxes the credit limit appropriately in return for a higher economic efficiency to balance the honesty and the efficiency [7-10]. To be specific, Gao et al. [7] raised a proposal by being against the integrated contract and auction design for secondary spectrum trading, in the premise of the dependence of spectrum in time and space while spectrum spatial reuse is not allowed. A two-level dynamic game framework is developed in [8], which can achieve optimal benefit when both 
secondary providers and secondary users are made dynamically by competition. Sung and Richard [9] analyzed both the cooperative and noncooperative games and listed the maximum thresholds of the benefits received.

On the other hand, to lower the overhead and time cost for spectrum pricing, pricing-based spectrum trading has also been widespread concerned either [11-14]. In [11], the authors considered that secondary users access idle spectrum in LTE architecture with a base-station-centric framework and introduced a pricing model based on benefit motivation. Zhong and Wang [12] analyzed the scheme of power and spectrum allocation to improve the energy efficiency through spectrum sharing. Moreover, Tan et al. [13] introduced the concept of user preference which obeys the uniform distribution to proceed spectrum pricing. Actually, a random and actual user's preference parameter should be a normal distribution. Thus, more deep research on this case should be extended to address the optimal spectrum pricing.

During dynamic spectrum access, most of current research studies extend their technical roadmaps with an underlying assumption that all the leased channels are homogeneous and undergo uniform interference. However, as the available spectrum to be leased is not predictable and always appearing in nonconsecutive bands, it may have various fading characteristics. Furthermore, due to secondary users' diverse positions in heterogeneous cells, even the same spectrum band does not mean the same utility for different users sometimes. Thus, heterogeneous spectrum and differential user selection on the spectrum deserve full investigation which is the main concern of this paper.

In this paper, we investigate how to price the spectrum especially when heterogeneous spectrum and stochastic secondary user's preference are under consideration. A concept of spectrum pool constituted by the idle bands to be leased is introduced to facilitate the following spectrum deal. Suffering from different levels of interference, these channels have various qualities. In this case, secondary users are supposed to select channels for usage based on their preference. It can be envisioned that a secondary buyer with sufficient capital or urgent demand for ideal QoS can pick a high-quality channel. A secondary spectrum customer will pick a high-quality channel for usage when its capital is ample or broadband is required to support essential service. We adopt Hotelling model which is proper to describe the product pricing issue in heterogeneous market. By analyzing the secondary user's preference parameter, an iterative algorithm for spectrum pricing is obtained after fixing the Nash equilibrium. Numerical results are further provided to evaluate how the pricing parameters affect the primary system's profits.

The remainder of this paper is organized as follows. We introduce the system model for dynamic spectrum pricing in Section 2. Section 3 gives the utility function and finds the Nash equilibrium. Furthermore, numerical results are supplied to analyze the performance of the pricing algorithm in Section 3. Finally, we conclude this paper in Section 4 .

\section{System Model}

Suppose the idle spectrum leased by the primary system forms a spectrum sharing pool, where the spectrum can be divided into many uniform channels for selling. Besides, the qualities of these channels are not homogeneous. For highquality channels, the secondary users suffer lower channel fading or adjacent channel interference. Thus, secondary users choose these channels according to their diverse preferences. The preference parameter is determined by the channel quality and channel price.

In this case, we cast the pricing problem into the model of duopoly competition where two suppliers, similar to highquality spectrum and low-quality spectrum, competing in prices and products, tend to attract more potential buyers which will finally lead to a dynamic balance. This idea became known among economists as the principle of minimum differentiation which was first proposed by Hotelling [15]. In fact, a mature market is always controlled by only few huge corporations which likely come into being a monopoly economy. Hotelling generalized Bertrand's model by considering different firms' locations in geographic space, and later this model was more often interpreted as a model of product differentiation which is an important feature of actual business.

To be specific, when applying Hotelling model to formulate an optimization problem, product price and product diversity should be considered during the process of building objective function. Besides, after solving the convex function and identifying the Nash equilibrium, the existence and rationality of the Nash equilibrium should be discussed.

Due to the spectrum quality diversity, secondary users should make rational choices on the channels since an ideal channel corresponds to a higher cost. On the other side, the primary system needs to set a proper pricing mechanism to optimize its profits according to the secondary customers' purchasing behaviors.

2.1. Utility Functions. In this paper, we consider that the spectrum trading is performed without auction activities. During the course, primary systems have no prior knowledge of the secondary customer's spectrum preference. In spectrum trading, the utility function of a secondary user can be expressed as

$$
U=\theta s-p,
$$

where $\theta$ denotes the secondary user's preference, $s$ denotes the channel quality, and $p$ is the channel price. In the spectrum sharing pool, there are two kinds of channels with diverse qualities to be chosen by secondary users as shown in Figure 1. We use $s_{1}$ to denote the high-quality channel and $s_{2}$ to denote the low-quality channel. Apparently, we have $s_{1}>s_{2}>0$. In this case, different channel qualities mean various transmission capacities. Furthermore, we suppose that the secondary user's preference parameter $\theta$ is subject to the normal distribution expressed as $g(\theta)$. $\theta$ locates in the region of $\left[\theta_{L}, \theta_{H}\right]$, and $\rho$ is the corresponding 


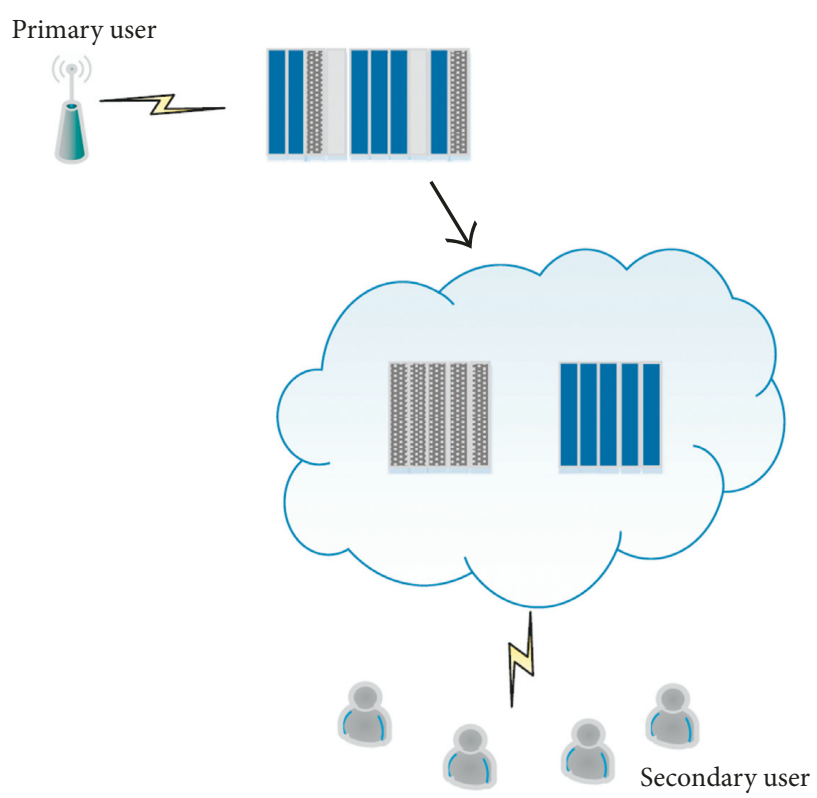

Idle channel

| Low-quality channel

| High-quality channel

FIgURe 1: Spectrum pool.

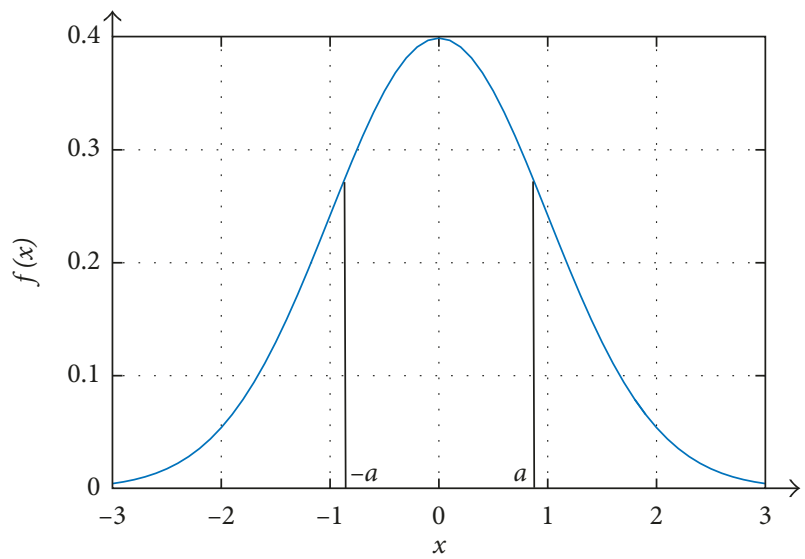

FIgURE 2: Standard normal distribution.

probability distribution function denoted as $\rho=G(\theta)$. We adopt $\theta_{0}$ to express the nonpreference parameters of cognitive users when there is no demand difference for the secondary customers between the high-quality channel and low-quality channel. Then, it can be calculated as $\theta_{0}=\left(p_{1}-p_{2}\right) /\left(s_{1}-s_{2}\right)$, where $p_{1}$ and $p_{2}$ represent the two kinds of channel prices. From the above analysis, when a secondary user's spectrum preference $\theta_{i}$ is higher than $\theta_{0}$, the user prefers to choose the high-quality channel. Otherwise, the user would rather prefer to choose the lowquality channel for purchasing.

Furthermore, a secondary user's selection preference is impacted by many factors, such as available budget, spectrum quality, and spectrum pricing denoted as (1). In fact,

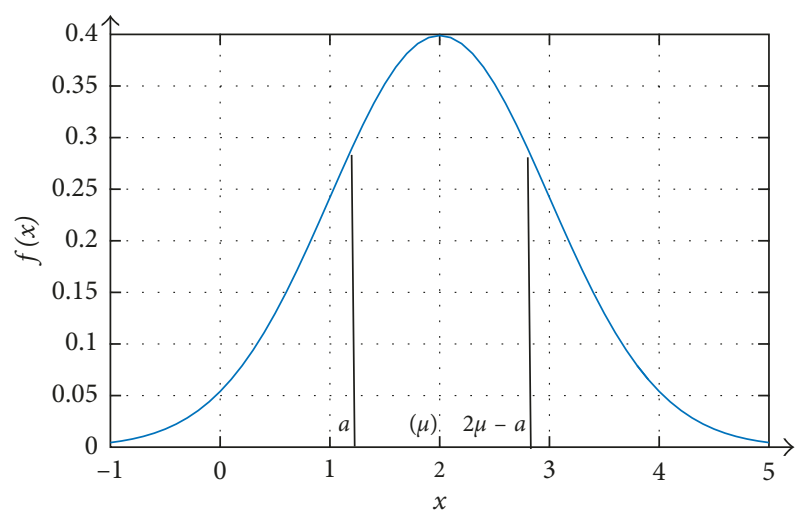

FIGURE 3: General normal distribution.

secondary user's preference shows the relation of demand and supply in current market. It is natural that a user's preference $\theta_{i}$ will increase when the spectrum pricing decreases or budget fund becomes abundant. In this situation, we consider when a user's preference locates at the region $\left[\theta_{1}, \theta_{2}\right]$, then the user will select a channel between highquality spectrum and low-quality spectrum with qualities $s_{1}$ and $s_{2}$, respectively.

Besides, in this case, we assume that the user's preference complies with normal distribution since a discrete random variable can be supposed to approximately obey normal distribution when the sample number is sufficient enough.

2.2. Spectrum Pricing. Secondary user's preference parameter is considered to be subject to the normal distribution for practical application. Figure 2 shows the density curve of the standard normal distribution. The probability density can be given as

$$
\varphi(x)=\frac{1}{\sqrt{2 \pi} e^{-x^{2} / 2}} .
$$

Then, the distribution function is

$$
f(x)=\int \varphi(x)=\frac{1}{2 \pi} \int_{-a}^{a} e^{-t^{2} / 2} d t .
$$

According to [16], $f(a)$ can be simplified as

$$
f(a)=\sqrt{1-e^{-a^{2} / 1.6058}} .
$$

Thus, the probability can be approximately calculated in the given region $[-a, a]$. Besides, according to [16], the conclusion obtained from (4) can also be applied to the case of general normal distribution as shown in Figure 3.

Furthermore, when the distribution mean is $\mu$, the probability calculated approximately in $[a, 2 \mu-a]$ is obtained as

$$
f(a)=\sqrt{1-e^{-(\mu-a)^{2} / 1.6058}} .
$$

As shown in Figure 4, the secondary customer whose preference parameter $\theta$ locates in $\left[\theta_{L}, \theta_{0}\right]$ will purchase lowquality channels. The user with the preference parameter $\theta \in\left[\theta_{0}, \theta_{H}\right]$ chooses a high-quality channel.

Then, we figure out the distribution probabilities for the two cases. Divide the part in red in Figure 4 into two parts, 


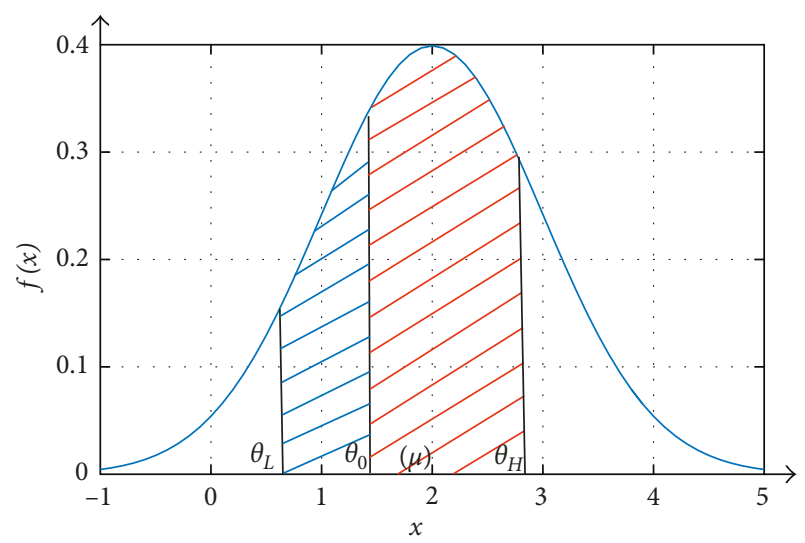

Figure 4: Divide the channels into two kinds of qualities.

wherein there are $\theta_{0}^{\prime}=\theta_{0}+\mu$ and $\theta_{H}-\theta_{H}^{\prime}=\mu$, as shown in Figure 5.

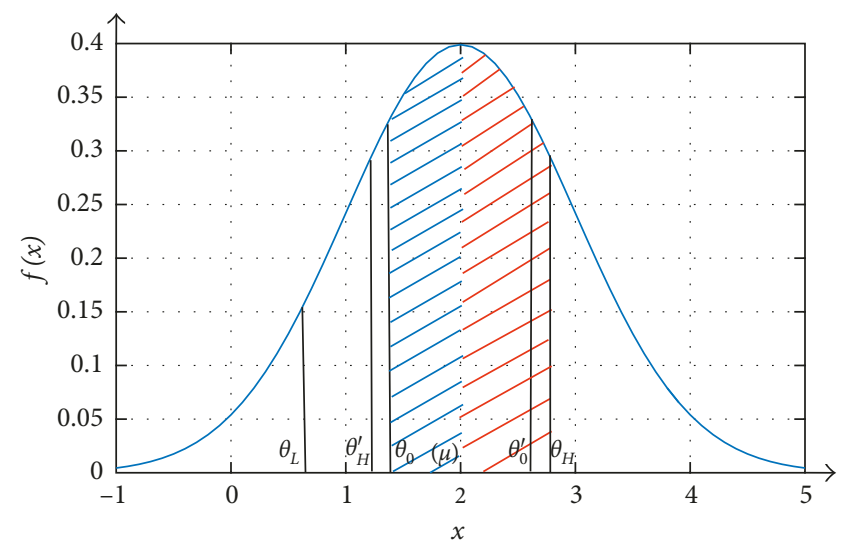

FIGURE 5: Divide the high-quality channels into two parts.

According to the corresponding regions of high-quality and low-quality channels, we have the probability densities as follows:

$$
\begin{gathered}
\varphi(H)=\frac{1}{2} \times\left(\sqrt{1-e^{-\left(\mu-\theta_{0}\right)^{2} / 1.6058}}+\sqrt{1-e^{-\left(\theta_{H}-\mu\right)^{2} / 1.6058}}\right), \\
\varphi(L)=\frac{1}{2} \times\left(\sqrt{1-e^{-\left(\mu-\theta_{L}\right)^{2} / 1.6058}}+\sqrt{1-e^{-\left(\mu-\theta_{0}\right)^{2} / 1.6058}}\right) .
\end{gathered}
$$

Besides, it is essential to take the opportunity cost of the primary system into account which means the primary system abandons the potential benefits in future to promote current spectrum trading. Thus, assume that the marginal cost of the primary user is related to the quality of the channel which can be expressed as $c_{i}=\alpha s_{i}(i=1,2)$, where $\alpha$ is the marginal factor. Formulating the problem by a Berland game model, the profit functions of systems $H$ and $L$ can be given as

$$
\begin{aligned}
& \pi_{H}\left(p_{1}, p_{2}\right)=N\left(p_{1}-\alpha s_{1}\right) \times \frac{1}{2} \times\left(\sqrt{1-e^{-\left(\mu-\theta_{0}\right)^{2} / 1.6058}}+\sqrt{1-e^{-\left(\theta_{H}-\mu\right)^{2} / 1.6058}}\right), \\
& \pi_{L}\left(p_{1}, p_{2}\right)=N\left(p_{2}-\alpha s_{2}\right) \times \frac{1}{2} \times\left(\sqrt{1-e^{-\left(\mu-\theta_{L}\right)^{2} / 1.6058}}+\sqrt{1-e^{-\left(\mu-\theta_{0}\right)^{2} / 1.6058}}\right),
\end{aligned}
$$

where $N$ is the number of secondary users. The nonpreference parameter $\theta_{0}$ is not fixed and changing in $\left[\theta_{L}, \theta_{H}\right]$. Besides, whether $\theta_{0}>\mu$ matters which will affect the deductions. The profits obtained above are subject to the situation when $\theta_{0}$ locates at the left side of $\mu$. Similarly, when $\theta_{0}$ locates at the right side of $\mu$, the corresponding profit functions can be deduced as

$$
\begin{aligned}
& \pi_{H}\left(p_{1}, p_{2}\right)=N\left(p_{1}-\alpha s_{1}\right) \times \frac{1}{2} \times\left(\sqrt{1-e^{-\left(\theta_{H}-\mu\right)^{2} / 1.6058}}+\sqrt{1-e^{-\left(\theta_{0}-\mu\right)^{2} / 1.6058}}\right), \\
& \pi_{L}\left(p_{1}, p_{2}\right)=N\left(p_{2}-\alpha s_{2}\right) \times \frac{1}{2} \times\left(\sqrt{1-e^{-\left(\mu-\theta_{L}\right)^{2} / 1.6058}}+\sqrt{1-e^{-\left(\theta_{0}-\mu\right)^{2} / 1.6058}}\right) .
\end{aligned}
$$

Based on the marginal utility functions, by taking derivatives of (7) and (8), we can achieve the iterative optimal channel pricing to be 


$$
\begin{aligned}
p_{1}^{(t+1)}= & p_{1}+\beta \times N \times \frac{1}{2} \times\left(\sqrt{1-e^{-\left(\mu-\theta_{0}\right)^{2} / 1.6058}}+\sqrt{1-e^{-\left(\theta_{H}-\mu\right)^{2} / 1.6058}}\right) \\
& +\beta \times N \times e^{-\left(\mu-\theta_{0}\right)^{2} / 1.6058} \times\left(2 \alpha-p_{1}^{(t)}\right) \times \frac{2 \mu-2 p_{1}^{(t)}-2 p_{2}^{(t)}}{6.4232 \sqrt{1-e^{-\left(\mu-\theta_{0}\right)^{2} / 1.6058}}}, \\
p_{2}^{(t+1)}= & p_{2}+\beta \times N \times \frac{1}{2} \times\left(\sqrt{1-e^{-\left(\mu-\theta_{L}\right)^{2} / 1.6058}}+\sqrt{1-e^{-\left(\mu-\theta_{0}\right)^{2} / 1.6058}}\right) \\
& +\beta \times N \times e^{-\left(\mu-\theta_{0}\right)^{2} / 1.6058} \times \frac{1}{6.4232} \times\left(2 p_{2}^{(t)}-2 p_{1}^{(t)}+2 \mu\right) .
\end{aligned}
$$

To be similar, we can achieve the optimal channel pricing when $\theta_{0}>\mu$.

As shown from the primary systems' utility functions expressed from (7) to (10), a key point to fix the final pricing solution is to ascertain that the utility function is consistent integrable. Then, we provide Theorem 1 to prove the essential condition.

Theorem 1. For the primary system's utility function $\pi_{i}$, if there is $\tau \geq 0$ satisfying $\sup _{i \in N} E\left(\pi_{i}\right)^{\tau}<\infty$, then we conclude $\pi_{i}$, $i \in N$, is consistent integrable.

Proof. When $\sup _{i \in N} E\left(\pi_{i}\right)^{\tau}<\infty$ can be met, we have the following inequality in the condition of $\chi>\chi_{\varepsilon}$ :

$$
P\left(\left|\pi_{i}\right| \geq \chi\right) \leq \frac{E\left|\pi_{i}\right|}{\chi} \leq \frac{\sup _{i \in N} E\left|\pi_{i}\right|^{\tau}}{\chi}<\delta_{\varepsilon}
$$

where $\pi_{i}$ denotes the spectrum preference of the secondary user $i$. Therefore, $E\left(\pi_{i}\right) I_{\left\{\left(\pi_{i}\right) \geq \chi\right\}} \leq \varepsilon$ holds for any $t \in N$, then we have

$$
\sup _{i \in t} E\left(\pi_{i}\right)^{\tau} I_{\left\{\left(\pi_{i}\right) \geq \chi\right\}} \leq \varepsilon, \quad \tau \geq 0,
$$

wherein for $\omega \in \Omega$,

$$
I_{A}(\omega)= \begin{cases}1, & \omega \in H, \\ 0, & \omega \in H^{C}:=\frac{\omega}{H} .\end{cases}
$$

Besides, for any $H \in \mathfrak{R}$ and $\chi>0$, we have

$$
\begin{aligned}
& \sup _{i \in N} E\left(\pi_{i}\right)^{\tau} I_{H} \\
& =\sup _{i \in N}\left(E\left(\pi_{i}\right)^{\tau} I_{H \cap\left\{\left(\pi_{i}\right)<\chi\right\}}+E\left(\pi_{i}\right)^{\tau} I_{H \cap\left\{\left(\pi_{i}\right) \geq \chi\right\}}\right) \\
& =\chi P(H)+\sup _{i \in N} E\left(\pi_{i}\right)^{\tau} I_{H \cap\left\{\left(\pi_{i}\right) \geq \chi\right\}} .
\end{aligned}
$$

If $\left\{\pi_{i}, i \in N\right\}$ is consistent integrable, $H=\Omega$ can be set which makes $\chi_{0}$ larger to obtain $\sup _{i \in N} E\left(\pi_{i}\right)^{\tau} I_{H \cap\left\{\left(\pi_{i}\right) \geq \chi_{0}\right\}}<1$, then we further have $\sup _{i \in N} E\left(\pi_{i}\right)^{\tau} \leq \chi_{0}+1<\infty$. Furthermore, for any $\varepsilon>0$, letting $\chi_{\varepsilon}$ large enough can guarantee $\sup _{i \in N} E\left(\pi_{i}\right)^{\tau} I_{H \cap\left\{\left(\pi_{i}\right) \geq \chi_{\varepsilon}\right\}}<\varepsilon / 2$. Let $\delta_{\varepsilon}=\varepsilon /\left(2 \chi_{\varepsilon}\right)$, for any $A \in \mathfrak{R}$, only if $P(H)<\delta_{\varepsilon}$, we can achieve

$$
\sup _{i \in t} E\left(\pi_{i}\right)^{\tau} I_{H} \leq \chi_{\varepsilon} P(H)+\frac{\varepsilon}{2}<\varepsilon
$$

Thus, the sufficient conditions can be satisfied to ensure the consistent integrability of the proposed utility function.

Besides, as the utility function may lead to multiple solutions, it is essential to remove the surplus solutions and find the optimal one for the rationality of the deduced Nash equilibrium. The proof for the existence of a pure Nash equilibrium is provided as follows.

Theorem 2. For a given utility function $f_{i}=\pi_{i}$ and strategy function $s_{i}=p_{i}$, a pure Nash equilibrium for the proposed pricing game algorithm exists.

Proof. Based on the theorem of Debreu's equilibrium existence [17], for the functions $s_{i}$ and $f_{i}$ given above, a pure strategy Nash equilibrium exists once the following sufficient conditions can be satisfied: (1) in limited Euclidean space, the strategy function $s_{i}$ is a nonempty and compact subset and (2) for a strategy combination $S, f_{i}$ is continuous and concave. First of all, we define $S: \Sigma \rightarrow \Sigma$ to be a Cartesian direct product for $s_{i}$, and $\Sigma_{i}$ is a simplex in dimension $\left(s_{i}\right) . s_{i}$ can be a compact subset in limited Euclidean space only when the condition that $s_{i}$ is symmetrically bounded and continuous functions is met. In order to prove that $s_{i}$ is symmetrically bounded, thus $\forall \varepsilon_{1}$, it requires certifying that $\exists \delta=\delta\left(\varepsilon_{1}\right)$ can make $\forall \psi \in F$ workable which can be

$$
\left|\psi\left(x_{1}\right)-\psi\left(x_{2}\right)\right|<\varepsilon_{1} \quad\left(\text { when } \rho\left(x_{1}, x_{2}\right)<\delta\right),
$$

where $\rho(x, y) \triangleq \max _{a \leq t \leq b}(x(t)-y(t))$ and $\psi\left(x_{i}\right) \Rightarrow s_{i}$. As the subset $\varepsilon_{1} / 3$ is finite $N\left(\varepsilon_{1} / 3\right)=\left\{\psi_{1}, \psi_{2}, \ldots, \psi_{n}\right\}$, then based on the characteristic of continuity, we have $\exists \delta=\delta(\varepsilon / 3)$. Therefore, in the condition of $\rho\left(x_{1}, x_{2}\right)$, we achieve the following inequality:

$$
\left|\psi_{i}\left(x_{1}\right)-\psi_{i}\left(x_{2}\right)\right|<\frac{\varepsilon_{1}}{3} \quad(i=1,2, \ldots, n) .
$$

As $\forall \psi \in F, \psi_{i} \in N(\varepsilon / 3)$ enables $d\left(\psi, \psi_{i}\right)<\varepsilon / 3$, we can obtain 


$$
\begin{aligned}
& \left|\psi(x)-\psi\left(x^{\prime}\right)\right| \\
& \leq\left|\psi(x)-\psi_{i}(x)\right|+\left|\psi_{i}(x)-\psi_{i}\left(x^{\prime}\right)\right|+\left|\psi_{i}(x)-\psi\left(x^{\prime}\right)\right| \\
& \leq 2 d\left(\psi, \psi_{i}\right)+\left(\psi_{i}(x)-\psi_{i}\left(x^{\prime}\right)\right)<\varepsilon_{1} \quad\left(\text { when } \rho\left(x, x^{\prime}\right)<\delta\right),
\end{aligned}
$$

where $d(u, v)=\max (u(x)-v(x))$. Besides, it is apparent that $s_{i}$ should be continuous and differentiable; thus, we can conclude that $s_{i}$ is a nonempty and compact subset in limited Euclidean space.

Furthermore, since the requirement for the concave function is that $f(t x+(1+t) y) \geq t f(x)+(1-t) f(y)$ and concave $f_{i}$ holds, we need the following equation for the high-quality channels:

$$
\pi_{i}^{\prime \prime}<0
$$

Obviously, proper parameter settings can satisfy this condition. Hence, we can conclude that the existence of a unique Nash equilibrium for our proposed algorithm is satisfied [18].

\section{Numerical Results}

In this section, numerical results are provided to testify the effects of the proposed pricing method. In dynamic access networks, we suppose that the idle spectrum is controlled by the licensed users. The secondary users who aim to access the spectrum must participate in the spectrum trading and pay for the cost to the primary systems. As the proposed pricing solution is an iterative algorithm, we thus give the initial spectrum pricing for two kinds of channels to be $s_{1}=2$, $s_{2}=1, N=100, \alpha=1, \mu=2.2$, and $\beta \in(0,0.028)$. The cognitive user's preference locates in $[1,3]$ which means $\theta_{L}=1$ and $\theta_{H}=3$. Furthermore, since the proposed pricing method is an iterative algorithm, we set the initial spectrum pricing for two kinds of qualities of channels as $p_{i}^{(0)}=0.01$. Then, we give the performances of the channel prices and system profits in the following tests.

In Figure 6, we give the performances of the channel prices obtained in this paper with different marginal factors $\alpha$. We can achieve from the figure that the optimal price of the high-quality channel is much higher than that of the lowquality channel. Furthermore, the channel pricing rises with increasing marginal factor $\alpha$, since higher marginal cost needs to be compensated for the primary system. We further obtain that the iterative algorithm converges very fast which will attain a stable value within 15 iterations. In the counterpart, Figure 7 gives the performances of the system profits under the optimal pricing with different marginal factors $\alpha$. It is apparent in Figure 7 that the system profits decrease with increasing marginal factor $\alpha$, which means the close relation between spectrum cost and system profit. Besides, it should be noted that the profit received on the high-quality channel overcomes that on the low-quality channel which is understandable since the primary system in nature expects to reap more profits through more excellent products.

Then, we give the presentations of channel prices and system profits under different preference distribution means

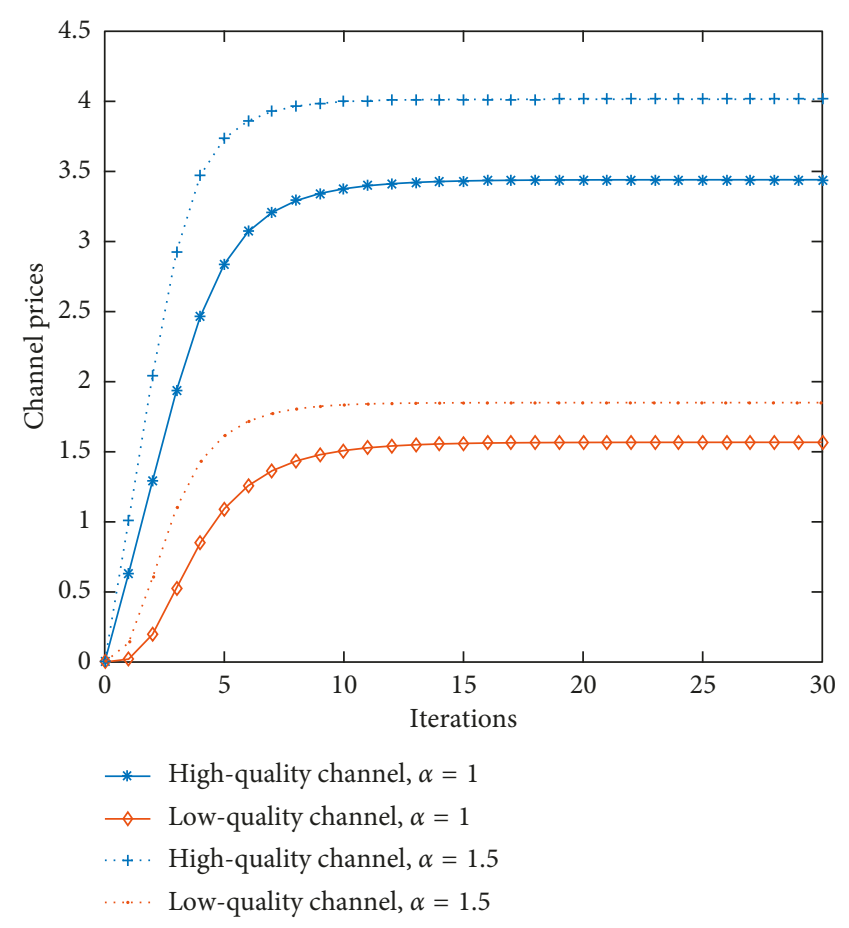

FIgURE 6: Channel prices with different marginal factors.

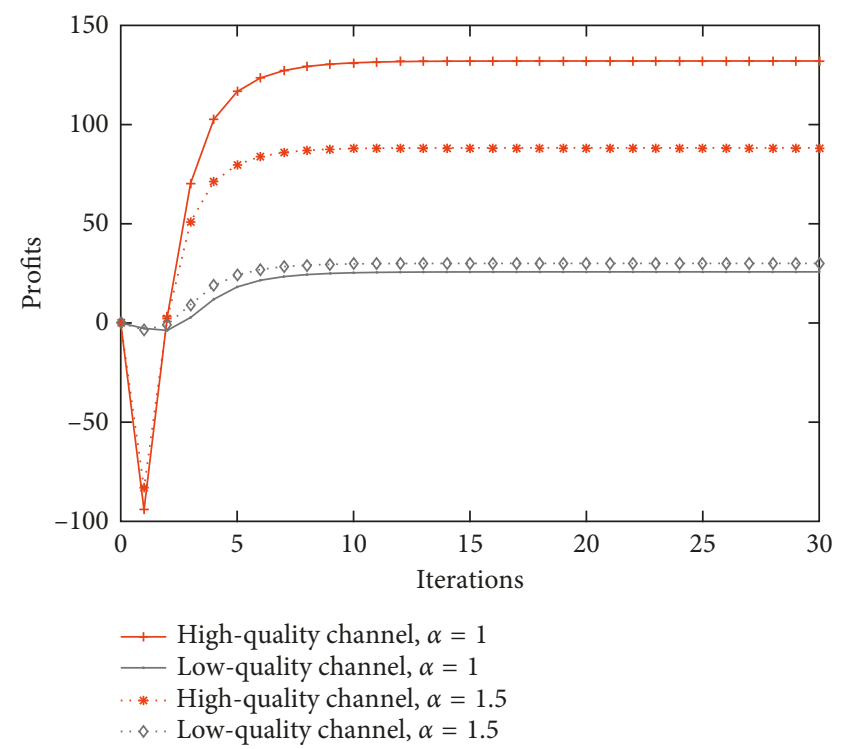

FIGURE 7: System profits with different marginal factors.

in Figures 8 and 9. Due to the cognitive user's preference factor $\theta$ locating in $[1,3]$, it would prefer a high-quality channel when $\theta$ approaches to 3 . Otherwise, it will choose a low-quality channel. As shown in Figure 8, there are two curves in response to one kind of color. We can obtain from the figure that the optimal channel price rises with the increasing distribution mean. It can be understood that the primary system considering the secondary customers with a higher preference factor would endure more expensive spectrum. As a result, the system profits upgrade with an 


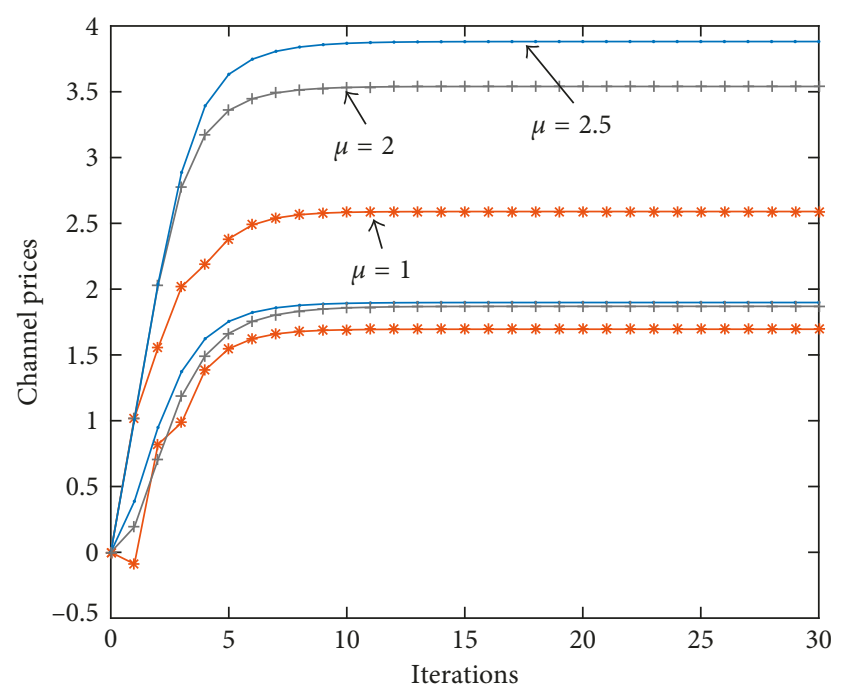

Figure 8: Channel prices with different distribution means.

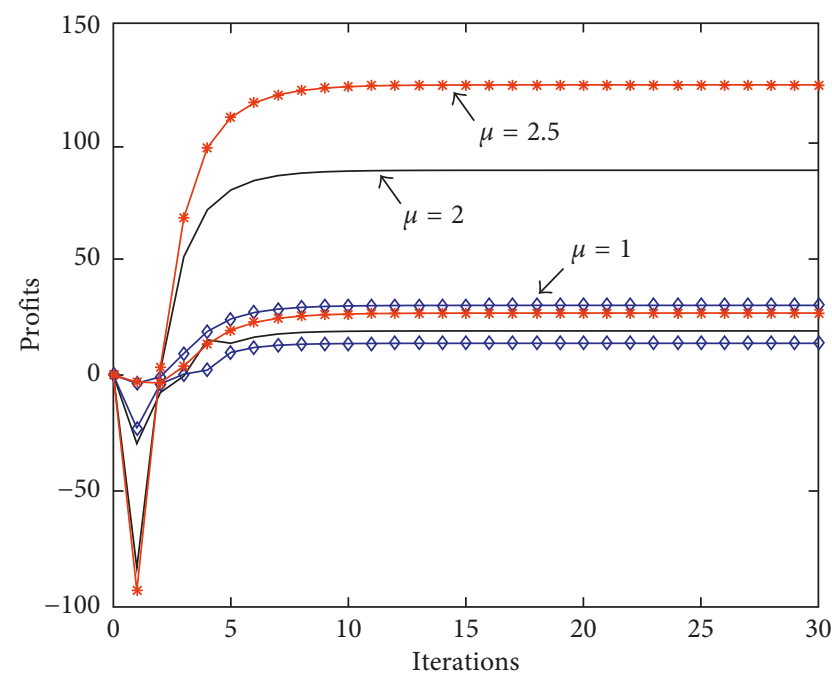

FIGURE 9: System profits with different distribution means.

increasing distribution mean. Meanwhile, the primary system can reap more profits when its high-quality spectrum attracts plenty of attention.

We can conclude from Figures 6-9 that the pricing curves for channel I to channel IV converge no more than 20 iterations. In fact, by choosing proper parameters, the proposed pricing algorithm can be guaranteed to converge at a fixed point.

Based on the pricing algorithm given in (11), the equations can be rewritten in the following matrix form:

$$
P^{k+1}=U^{-1} W P^{k}+U^{-1} b, \quad k=0,1,2, \ldots
$$

According to [19], if $\rho\left(U^{-1} W\right)<1$ holds, the iterative algorithm $p^{k}$ will be convergent. Based on the proposed pricing algorithm, we can easily achieve the Jacobi matrix and the corresponding maximal eigenvalue. Hence, by properly setting the algorithm's parameter, we can ensure

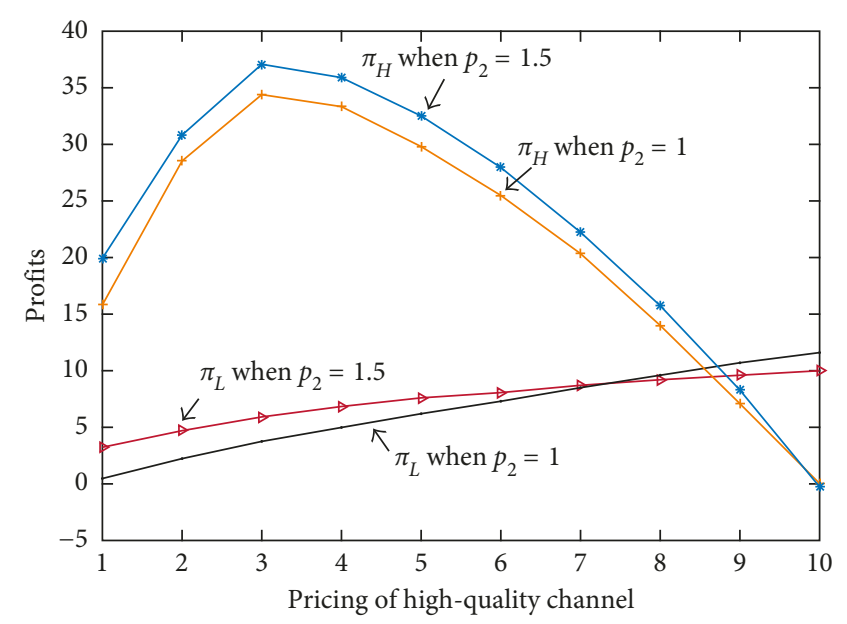

Figure 10: System profits with changing channel pricing.

$\rho\left(U^{-1} W\right)<1$. In addition, the oscillating point appears when $\rho\left(U^{-1} W\right)=1$.

At last, we testify the performances of system profits with different channel prices as shown in Figure 10. We can obtain from the figure that the system profits of the highquality channels do not grow continually with the increasing price of high-quality channel. Similar to the conclusions we deduced before, the utility functions of the system profits are convex with respect to corresponding channel pricing. Thus, the system profits of the high-quality channels can attain a maximum. Besides, the performances of low-quality channel's profits differ from that of the high-quality channel as the argument in $x$-axis is the pricing of high-quality channel. On the other hand, the increase of the high-quality channel's pricing will lead to the loss of potential customers to the high-quality channel which in turn improves the lowquality channel's profits.

\section{Conclusion}

In this paper, we investigate how to price the heterogeneous spectrum in condition of secondary users' stochastic selection preferences. The main contribution of this paper lies in that we introduce a Hotelling game model to formulate and address the differential spectrum pricing. In the proposed model, we assume that the idle spectrum is collected and leased to potential secondary users centrally so that a centralized spectrum pricing can be carried out by the primary system. Various qualities of idle spectrum constitute a spectrum pool in which the bands are divided into numbers of uniform channels for leasing. It is foreseen that the high-quality channels can incur more profits for the primary system by offering a high price. On the other hand, a preference factor is introduced to describe the secondary user's selection tendency on the channels. We analyze the impact of secondary users' preference on spectrum trading and propose an iterative algorithm for pricing. Proofs of the integrability of the utility function and existence of Nash equilibrium used in the proposal are also given. Numerical results are provided to testify the performances of the 
proposed optimal spectrum pricing and the corresponding system profits as a result.

\section{Conflicts of Interest}

The authors declare that they have no conflicts of interest.

\section{Acknowledgments}

This work was supported by the National Natural Science Foundation of China under Grant no. 51404211.

\section{References}

[1] N. Zhao, F. R. Yu, H. Sun, and M. Li, "Adaptive power allocation schemes for spectrum sharing in interferencealignment-based cognitive radio networks," IEEE Transactions on Vehicular Technology, vol. 65, no. 5, pp. 3700-3714, 2016.

[2] F. Li, X. Tan, and L. Wang, "An uplink power control algorithm using traditional iterative model for cognitive radio networks," Journal of Central South University, vol. 19, no. 10, pp. 2816-2822, 2012.

[3] C. Yang, J. Li, and A. Anpalagan, "Hierarchical decisionmaking with information asymmetry for spectrum sharing systems," IEEE Transactions on Vehicular Technology, vol. 64, no. 9, pp. 4359-4364, 2015.

[4] X. Liu, F. Li, and Z. Na, "Optimal resource allocation in simultaneous cooperative spectrum sensing and energy harvesting for multichannel cognitive radio," IEEE Access, vol. 5, pp. 3801-3812, 2017.

[5] F. Li, X. Tan, and L. Wang, "A new game algorithm for power control in cognitive radio networks," IEEE Transactions on Vehicular Technology, vol. 60, no. 9, pp. 4384-4392, 2011.

[6] X. Liu, M. Jia, and X. Tan, "Threshold optimization of cooperative spectrum sensing in cognitive radio network," Radio Science, vol. 48, no. 1, pp. 23-32, 2013.

[7] L. Gao, J. Huang, Y. Chen, and B. Shou, "An integrated contract and auction design for secondary spectrum trading," IEEE Journal on Selected Areas in Communications, vol. 31, no. 3, pp. 581-592, 2013.

[8] K. Zhu, N. Dusit, P. Wang, and Z. Han, "Dynamic spectrum leasing and service selection in spectrum secondary market of cognitive radio networks," IEEE Transactions on Wireless Communications, vol. 11, no. 3, pp. 1136-1145, 2012.

[9] C. H. Sung and J. L. Richard, "Secondary spectrum tradingauction-based framework for spectrum allocation and profit sharing," IEEE/ACM Transactions on Networking, vol. 21, no. 1, pp. 176-189, 2012.

[10] W. Wang and X. Liu, "List-coloring based channel allocation for open-spectrum wireless networks," in Proceedings of 2005 IEEE 62nd Vehicular Technology Conference 2005, pp. 690-694, Dallas, TX, USA, 2005.

[11] D. Soumitra, P. Shalini, and Y. Halim, "Secondary user access in LTE architecture based on a base-station-centric framework with dynamic pricing," IEEE Transactions on Vehicular Technology, vol. 62, no. 1, pp. 284-296, 2012.

[12] W. Zhong and J. Wang, "Energy efficient spectrum sharing strategy selection for cognitive MIMO interference channels," IEEE Transactions on Signal Processing, vol. 61, no. 14, pp. 3705-3717, 2013.

[13] X. Tan, Y. Liu, and S. Wei, "Game-based spectrum allocation in cognitive radio networks," Journal of South China University of Technology, vol. 38, no. 5, pp. 22-38, 2010.
[14] Y. Liu, M. Jiang, X. Tan, and F. Lu, "Maximal independent set based channel allocation algorithm in cognitive radios," in Proceedings of IEEE Youth Conference on Information, Computing and Telecommunication 2009, pp. 78-81, Beijing, China, 2009.

[15] H. Hotelling, "Stability in competition," Economic Journal, vol. 39, no. 153, pp. 41-57, 1929.

[16] M. Li, Research of Cognitive Radio Spectrum Allocation Algorithm Based on Graph Theory, Southwest Jiaotong University, Chengdu, China, 2012.

[17] G. Debreu, "A social equilibrium existence theorem," Proceedings of the National Academy of Sciences, vol. 38, no. 10, pp. 886-893, 1952.

[18] A. M. Colell, M. D. Whinston, and J. R. Green, Microeconomic Theory, Oxford University Press, Oxford, UK, 1995.

[19] Y. Saad, Iterative Methods for Sparse Linear System, Society for Industrial and Applied Mathematics, Philadelphia, PA, USA, 2nd edition, 2003. 


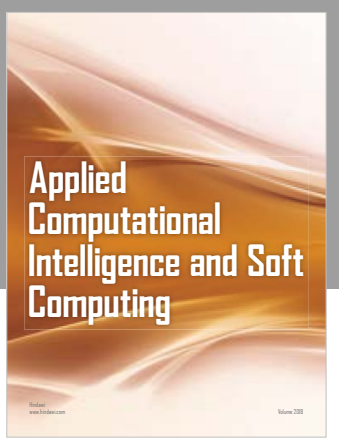

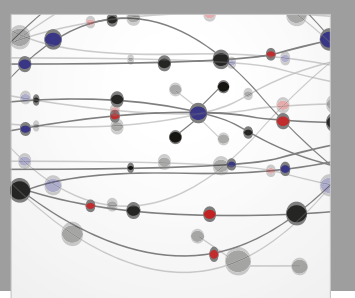

The Scientific World Journal
Submit your manuscripts at

Computing
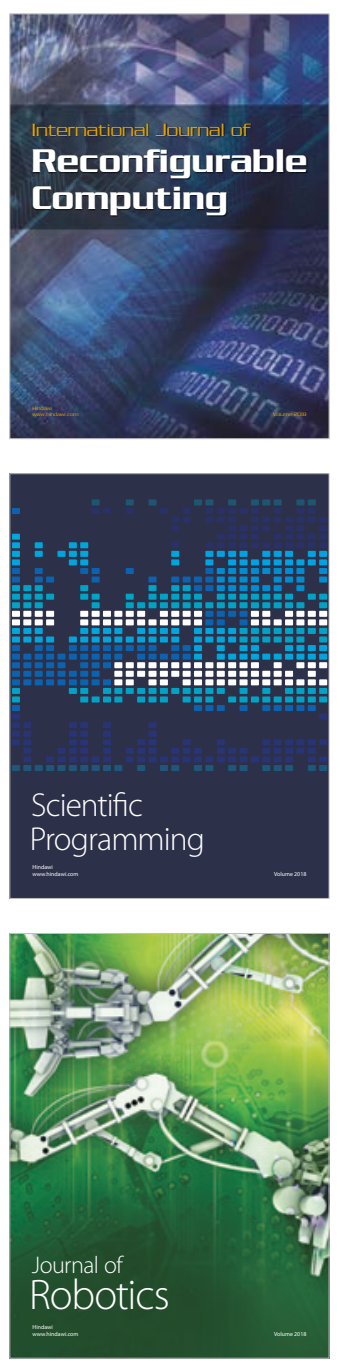

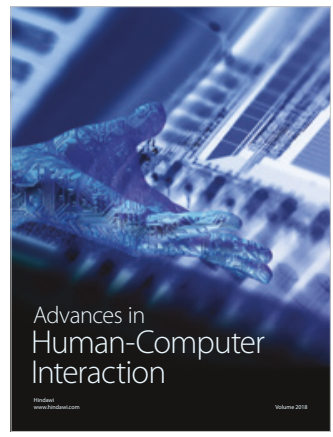

Human-Compute

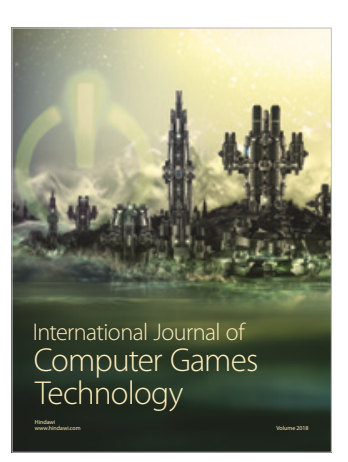

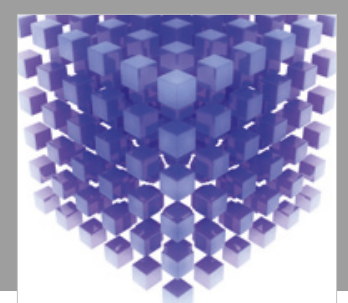

Mathematical Problems in Engineering

\section{Engincering}
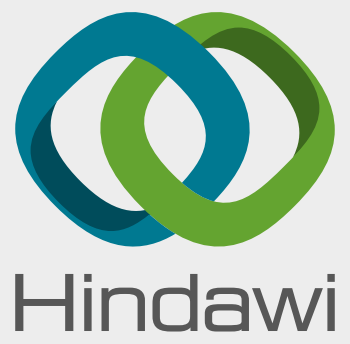

www.hindawi.com
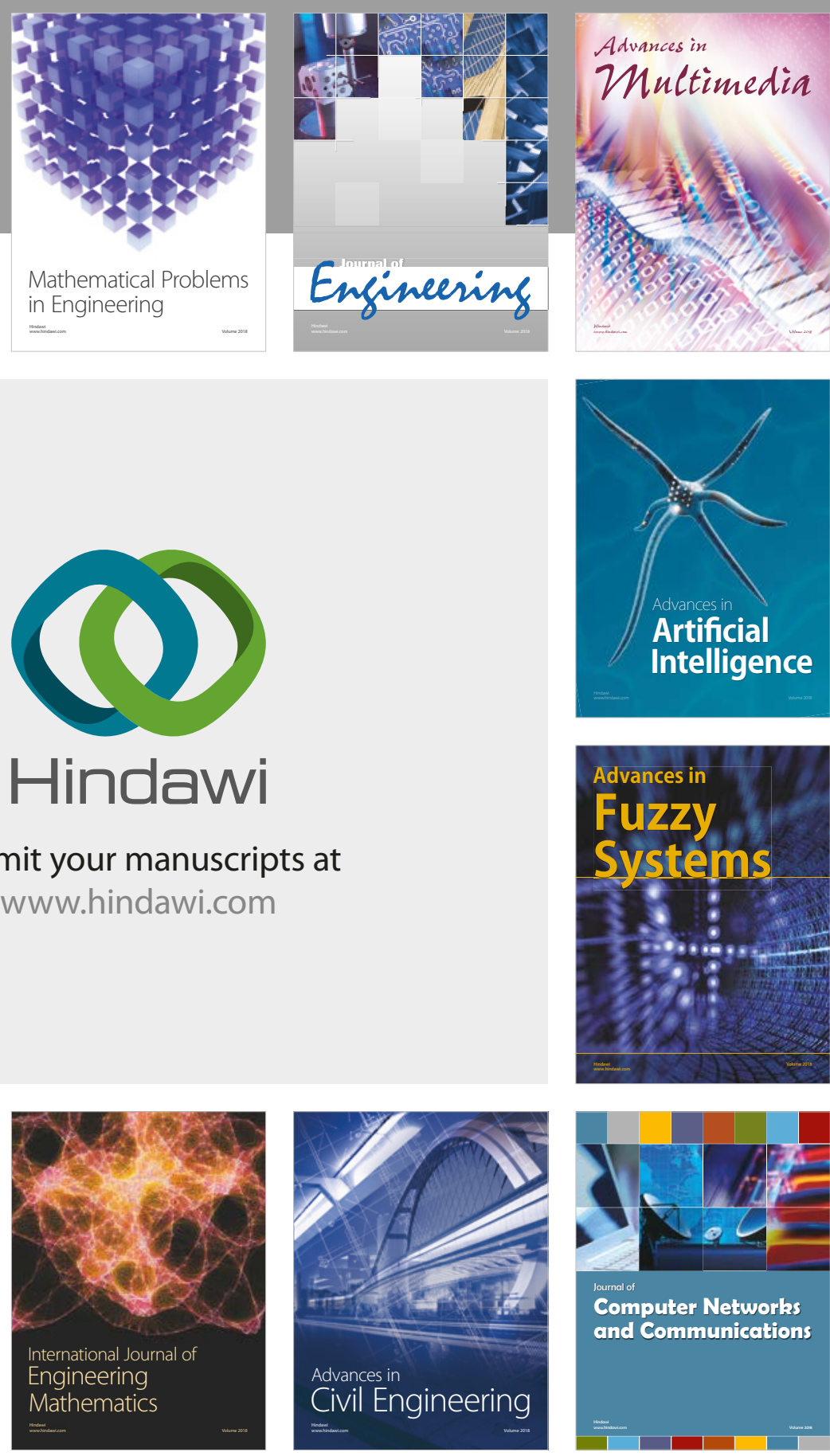

Computer Networks and Communications

Multimedia
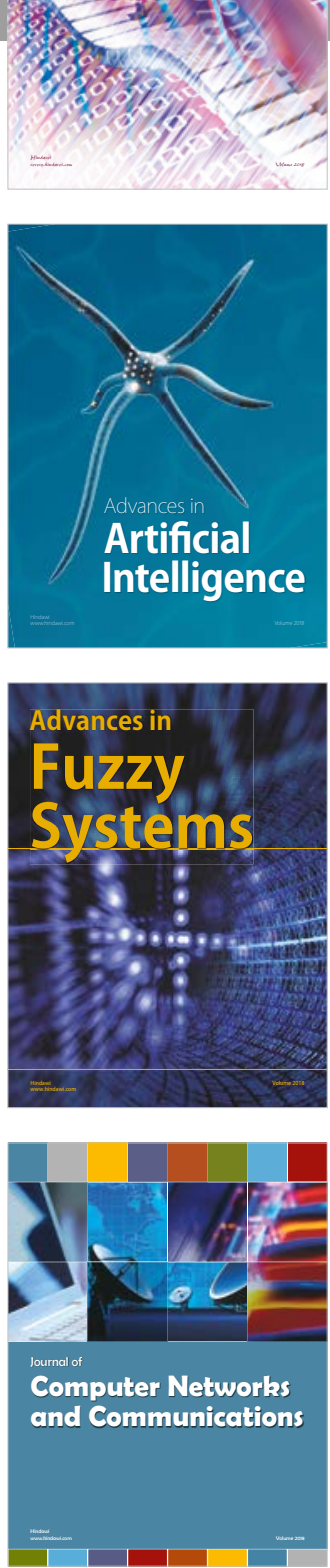

Advances in

Modelling \&

Simulation

in Engineering

interaction

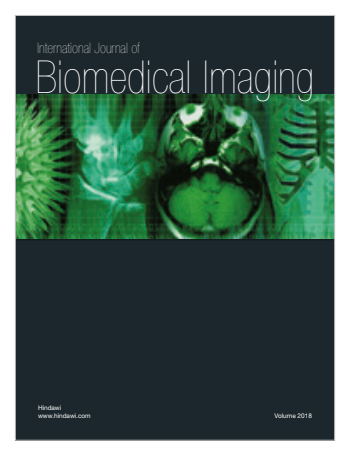

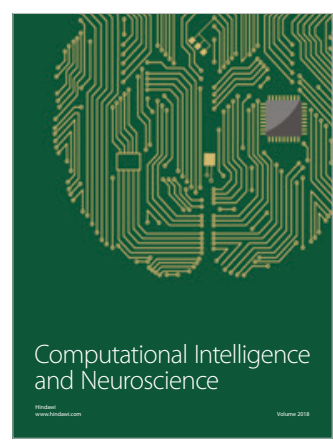

